\title{
Utilisation des cellules au silicium amorphe pour la mesure du rayonnement photosynthétiquement actif $(400-700 \mathrm{~nm})$
}

\author{
M. Chartier, P. Bonchretien, J.M Allirand et G. Gosse \\ Station de Bioclimatologie, INRA, 78850 Thiverval-Grignon, France
}

(reçu le 6-10-1988, accepté le 6-12-1988)

Résumé - La simulation et la confrontation expérimentale lus réponses des cellules au silicium amorphe par rapport à celles des capteurs existants montrent l'intérêt des cellules au silicium amorphe pour la mesure du rayonnement PAR (exprimé en densité de flux de photons) incident, réfléchi et transmis sous un couvert végétal.

cellules au silicium amorphe - rayonnement photosynthétiquement actif - bioclimatologie

Summary - Amorphous silicon cells for the measurement of photosynthetically active radiation. Numerical simulation and experimental measurements from amorphous silicon cells in comparison with these now used in ecophysiology illustrate the interest of amorphous silicon cells for the measurement of PAR incident, reflected, and transmitted below the canopy.

amorphous silicon cells - photosynthetically active radiation - bioclimatology

\section{Introduction}

Le flux de photons correspondant à la gamme du rayonnement photosynthétiquement actif (400-700 nm) (nous adopterons par la suite le sigle PAR, défini par les Anglo-Saxons) présente un intérêt certain en écophysiologie et plus particulièrement pour les études concernant la photosynthèse et la croissance. Depuis la définition qu'en a faite McCree (1972), différents auteurs et industriels se sont efforcés de réaliser des capteurs permettant d'en faire une mesure précise. Les capteurs de la société Licor ${ }^{\star}$ peuvent être considérés actuellement comme les capteurs de référence.

En matière d'écophysiologie, les problèmes posés par la mesure simple mais fiable de ce flux de photons PAR concernent d'une part la mesure du rayonnement incident moyen (en conditions d'éclairage naturel et/ou artificiel) et, d'autre part,

* Licor, Box 4425, Lincoln, Nebraska, 68504 USA. la mesure du rayonnement transmis et/ou absorbé par un couvert végétal. L'objet de cette note est de tester la possibilité d'utiliser des cellules au silicium amorphe pour mesurer le rayonnement PAR transmis sous une culture. Compte tenu de son faible coût, lié à l'absence d'utilisation de filtres, et de la possibilité d'obtenir des cellules de tailles différentes permettant de s'adapter à différentes structures de végétation, ce type de cellule présente alors de nombreux avantages par rapport aux capteurs existants sur le marché.

\section{Matériel et Méthodes}

Les photopiles au silicium amorphe (dénommées ensuite Slam) employées sont des cellules standard de la société Solems ${ }^{\star}$ et elles ont été testées par rapport au capteur Licor (LI 190S), classiquement utilisé en écophysiologie pour la mesure du PAR exprimé en

\footnotetext{
* Solems, Zl Les Glaises, 3, rue Léon-Blum, 91120 Palai-
} seau, France 
nombre de photons. Ces cellules Licor sont des cellules au silicium cristallin munies des filtres interférentiels adéquats (Fig. 1). Bien que ces derniers capteurs ne puissent être considérés comme des étalons primaires, ils représentent néanmoins ce qui s'en approche le plus actuellement. teurs:

Les tests ont porté sur deux grands types de fac-

- ceux concernant la réponse du capteur dans nos conditions d'utilisation, et plus particulièrement les variations de la réponse en fonction du spectre de la source;

- ceux concernant la réponse intrinsèque du capteur et ses conditions de montage : test de l'effet cosinus et de l'action de la température, courant de saturation, linéarité du signal, vieillissement...

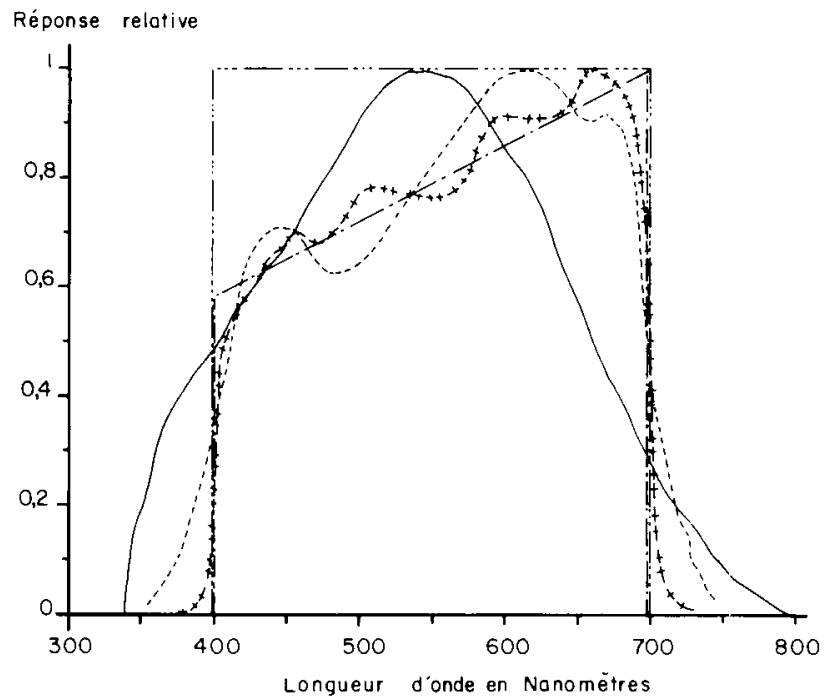

Fig. 1. Réponse relative spectrale en énergie des différents capteurs théoriques $(-\ldots-$ mesure en énergie, $-\cdots-\cdots$ mesure en nombre de photons, - . - selon McCree) ou existants (- Solems, +++ Licor) analysés dans cette étude.

\section{Résultats}

Analyse des paramétres liés à la réponse du capteur dans nos conditions d'utilisation

A partir de la réponse spectrale fournie par le constructeur (Fig. 1) et des courbes de sensibilité spectrale d'un capteur mesurant le PAR en énergie, en nombre de photons ou ayant la courbe de réponse idéale définie par McCree (1972) (Fig. 1), il est possible de simuler, pour des sources lumineuses présentant des spectres différents, la réponse théorique de la cellule testée.

Cette simulation a été faite d'une part pour des spectres de lampes classiquement utilisées en conditions contrôlées : tube fluorescent, lampe au tungstène, lampe aux iodures métalliques et lampe à vapeur de sodium HP (Tableau I) et, d'autre part,pour des spectres de rayonnement solaire correspondant à des conditions extrêmes d'ennuagement (ciel clair et couvert) ainsi que pour un spectre de rayonnement mesuré sous un couvert végétal (Varlet-Grancher, 1982).

Le Tableau I illustre l'évolution des rapports théoriques cellule Slam et cellule Licor par rapport à la courbe de sensibilité définie par McCree pour l'ensemble des spectres analysés. En conclusion, bien que présentant une réponse spectrale s'écartant quelque peu de la courbe idéale, la cellule Slam peut en théorie être utilisée pour la mesure du flux de photons PAR. En conditions naturelles, la variabilité simulée du rapport est du même ordre de grandeur que celle obtenue avec des cellules Licor; il est en outre possible d'améliorer ce résultat en munissant ce capteur d'un filtre arrêtant les UV. En conditions contrôlées, les défauts observés au niveau du spectre de réponse de la cellule Slam donnent des écarts sensibles dans le cas des lampes au tungstène présentant un spectre croissant en fonction de la longueur d'onde dans le visible. Pour les lampes les plus utilisées en chambre de culture (tube fluo, lampe à vapeur de sodium, lampe aux iodures métalliques), les cellules au silicium amorphe peuvent aussi être utilisées, munies d'un filtre UV de préférence.

Tableau I. Evolution des rapports simulés Slam/Mc:Cree et Licor/McCree pour les différentes sources lumineuses testées.

Références des lampes utilisées : - lampes sodium haute pression, Thorn Emi, cf SON-T 400W; - tube fluo, Sylvania Luxline-ES, blanc chaud de luxe 58W; - lampe tungstène, halogène $250 \mathrm{~W}$; - lampe aux iodures métalliques, Philips HPI-T 400W.

Caractéristiques des spectres solaires d'après Varlet-Grancher (1982) : (1) $h=46^{\circ} D / G=0,35$, (2) $h=64^{\circ} D / G=0,23$, (3) $h>45^{\circ} D / G=1$, (4) sous un couvert de maïs $(I F=3,4)$.

\begin{tabular}{|c|c|c|c|c|c|c|c|c|}
\hline & \multicolumn{4}{|c|}{ Sources artificielles } & \multicolumn{4}{|c|}{ Rayonnement solaire } \\
\hline & $\begin{array}{l}\text { Lampe } \\
\text { Sodium }\end{array}$ & $\begin{array}{l}\text { Tube } \\
\text { fluo }\end{array}$ & $\begin{array}{l}\text { Lampe } \\
\text { tungstène }\end{array}$ & $\begin{array}{l}\text { Lampe } \\
\text { iod. métal. }\end{array}$ & (1) & (2) & (3) & (4) \\
\hline Slam/McCree & 0,952 & 1,005 & 0,820 & 1,054 & 0,978 & 0,992 & 0,995 & 1.002 \\
\hline Slam/McCree + UV & 0,938 & 0,948 & 0,800 & 1,038 & 0,931 & 0,941 & 0,941 & 0,941 \\
\hline Lioor/McCree & 0,930 & 0,977 & 0,947 & 0,964 & 0,970 & 0,969 & 0,972 & 0,967 \\
\hline
\end{tabular}


Ces conclusions peuvent se généraliser aux autres sources lumineuses présentant leur maximum d'émission entre 500 et $600 \mathrm{~nm}$, ce qui est le cas du rayonnement solaire, des lampes à vapeur de sodium ou des tubes fluorescents; un coefficient d'étalonnage spécifique serait à envisager pour des spectres présentant leur maximum au delà de $700 \mathrm{~nm}$, ce qui est le cas des lampes au tungstène ou des halogènes (beaucoup moins utilisées en expérimentation).

Dans un second temps, les résultats de cette simulation ont été confrontés à l'expérimentation; les tests comparatifs entre les cellules Slam (sans filtre UV) et les cellules type Licor ont été réalisés, en conditions contrôlées pour les lampes utilisées dans la précédente simulation et en conditions naturelles pour des types de ciel clair et couvert. Le Tableau II donne l'évolution du rapport Slam/Licor pour l'ensemble de ces conditions. L'accord entre la simulation et la mesure est très bon, ce qui confirme les conclusions développées précédemment pour l'utilisation de ce type de capteur.

Analyse des conditions de montage et d'utilisation

D'autres caractéristiques physiques de ce capteur ont été testées, notamment : le temps de réponse, le coefficient de température, l'effet d'un diffuseur et l'effet d'inclinaison :

- le temps de réponse est du même ordre de grandeur que celui des cellules au silicium cristallin (type Licor), soit $0,2 \sec$ pour $66 \%$ de la réponse ;

- le coefficient de température est un peu plus élevé que pour une cellule au silicium cristaliin (Fig. 2), ce qui conduit à une erreur inférieure à $0,2 \%{ }^{\circ} \mathrm{C}$ entre -5 et $40^{\circ} \mathrm{C}$, valeur supérieure à celle du capteur Licor, mais acceptable pour toute application dans le secteur agronomique ;

- le Tableau III illustre l'effet de l'inclinaison de la source par rapport au capteur; cet effet est du

Tableau II. Evolution des rapports mesurés Slam/cellule Lioor (en valeurs relatives) en fonction du type d'éclairage (naturel, artificiel).

\begin{tabular}{lll} 
Type d'éclairage & Slam/Licor & $\sigma$ \\
\hline Lampe iod. métal & 1,278 & 0,005 \\
Lampe sodium & 1,264 & 0,005 \\
Lampe fluo & 1,332 & 0,004 \\
Lampe tungstène & 1,243 & 0,004 \\
Diverses conditions & & \\
naturelles & 1,273 & 0,013 \\
\hline
\end{tabular}

même ordre de grandeur que pour les cellules Licor (effet négligeable pour des inclinaisons supérieures à $10^{\circ}$ ). En conditions naturelles, la réponse du capteur peut encore être améliorée par l'addition d'un diffuseur neutre (Fig. 3), ce qui permet en plus d'éviter le risque de saturation aux très forts éclairements.

Compte tenu de la notice technique de la société Solems (Anonyme, 1987) et du domaine d'utilisation envisagé, on peut insister sur les points critiques suivants :

- la mesure du courant de court-circuit permet une très bonne linéarité entre 0 et 1500 $\mu$ moles $/ \mathrm{m}^{2} / \mathrm{sec}$ (courant de 7 à $9 \mathrm{~mA}$ par $\mathrm{cm}^{2}$ de cellule pour $1500 \mu$ moles), le léger effet de saturation observé à des éclairements supérieurs (Fig. 3) peut être éliminé par l'emploi d'un diffuseur (type altuglass translucide). Un signal en tension peut être mesuré en faisant débiter la cellule dans une résistance, afin de conserver la linéarité du signal et compte tenu de la courbe courant-tension de la cellule, la valeur de cette résistance sera comprise entre 5 et 20 ohms;

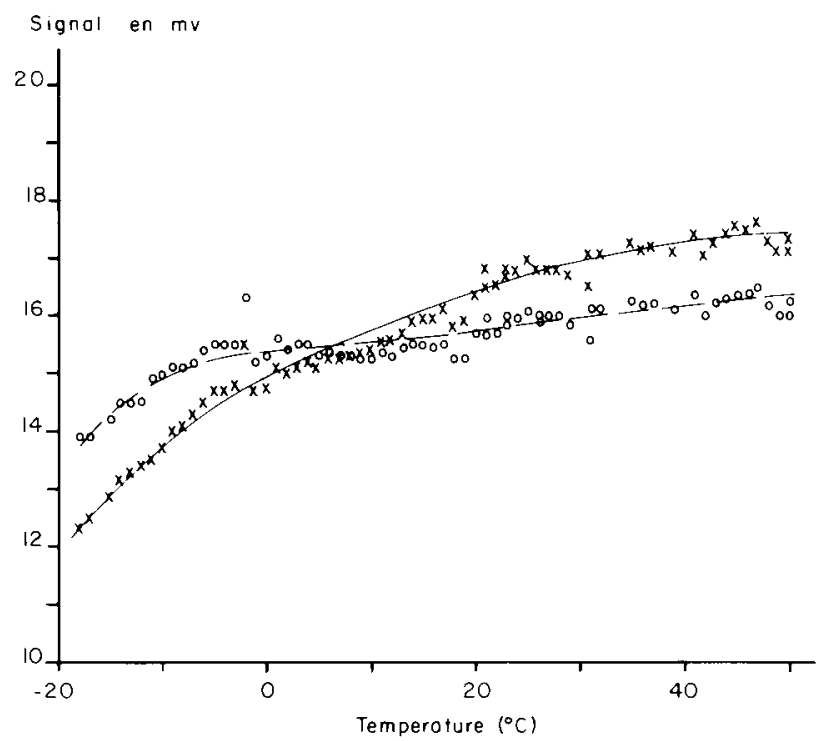

Fig. 2. Effet de la température sur la réponse des photopiles au silicium amorphe (Solems, $x$ ) et cristallin (Licor,o).

Tableau III. Effet de l'inclinaison de la source par rapport au capteur (équipé d'un diffuseur) sur le rapport Slam/Licor.

\begin{tabular}{cl} 
Angle d'incidence & Slam/Licor \\
\hline 90 & 1 \\
60 & 0,998 \\
45 & 0,996 \\
30 & 0,982 \\
10 & 0,965
\end{tabular}




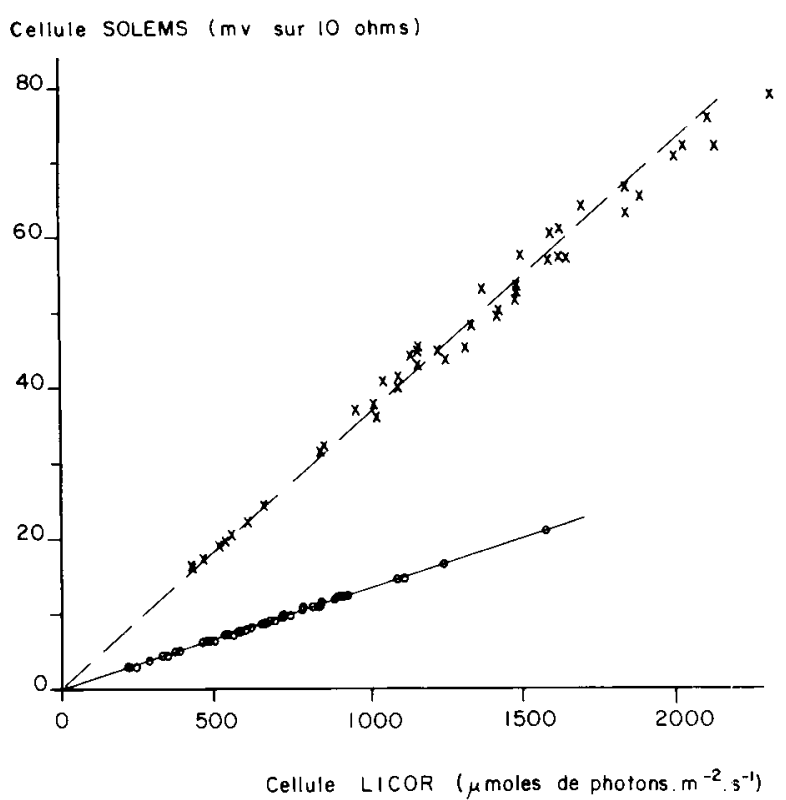

Fig. 3. Comparaison d'une cellule Slam et d'une cellule Licor dans les conditions naturelles, avec (o,jour 67) et sans (x,jour 68) diffuseur.

- ce type de cellule est sujet au vieillissement (diminution du signal de sortie). Deux causes sont possibles : a) vieillissement en début d'utilisation, ce point peut être résolu par une insolation au laboratoire ou chez le constructeur à un fort éclairement pendant un semaine, le vieillissement observé par la suite est négligeable dans le cadre des applications envisagées; b) vieillissement dû à une utilisation en atmosphère très humide, les cellules peuvent être livrées encapsulées (prix en augmentation importante, mais restant concurrentiel avec les capteurs existants), ou bien l'utilisation en l'état limitera leur durée de vie à quelques mois, ce qui reste encore satisfaisant pour de nombreuses applications en écophysiologie;

- les dimensions unitaires des cellules - ce dernier point est important pour l'adaptation des capteurs à la géométrie des couverts étudiés. La cellule utilisée pour ce test avait pour dimensions $16 \times 16 \times 2 \mathrm{~mm}$, les dimensions vont actuellement de $9 \times 16 \mathrm{~mm}$ à $100 \times 100 \mathrm{~mm}$ pour des celluies monodiodes. Cette plage de variation permet ainsi d'aborder la mesure du rayonnement transmis pour des couverts allant des cultures fourragères (fortes densités, faible écartement des rangs) à des cultures du type maïs ou tournesol (faible densité et fort écartement des rangs);

- la comparaison des réponses d'un lot de cellules issues d'une même fabrication présente une variabilité très faible (inférieure à $3 \%$ ); ceci permet d'envisager des montages en série ou en parallèle de cellules monodiodes.

\section{Intérêt pour la mesure du rayonnement transmis}

L'intérêt majeur de ce type de capteur réside dans l'obtention d'une mesure de PAR en l'absence de filtres interférentiels coûteux. Dans ces conditions, il devient possible de réaliser des capteurs modulaires, de taille et de géométrie adaptées à la stucture du peuplement végétal étudié. En effet, une bonne mesure du rayonnement PAR transmis à la base d'un couvert végétal requiert une méthodologie différente selon que l'on s'adresse à un couvert homogène ou anisotrope horizontalement. Dans le cas d'un couvert homogène horizontalement, il est possible de réaliser électriquement une intégration spatiale du résultat (type capteurs linéaires), à condition que la taille du capteur élémentaire soit inférieure à la tache de soleil élémentaire. Par contre, dans le cas de structures anisotropes, (cas des cultures en phase d'implantation), il est indispensable de traiter le signal élémentaire selon sa position dans le couvert (entre ou sous les lignes pour schématiser).

Dans les deux cas, la contrainte liée au nombre et à la taille élémentaire du capteur existe; elle peut être éliminée en utilisant des cellules au silicium amorphe.

\section{Conclusion}

Bien que cette étude métrologique soit faite par rapport à un étalon secondaire (les cellules Licor), les résultats obtenus montrent que les cellules au silicium amorphe présentent des avantages importants (qualité de l'information mesurée, faible coût et adaptabilité géométrique du capteur final...). Ces avantages permettent de mesurer avec précision et fiabilité le rayonnement PAR transmis et absorbé par une culture, celui-ci étant un des éléments déterminants pour l'analyse de la croissance d'un couvert végétal.

\section{Références}

Anonyme (1987) Utilisation des Photopiles au Silicium Amorphe. Guide Technique à I'Usage des Bureaux d'Etudes. Ed. Solems

McCree K.J. (1972)Test of current definitions of photosynthetically active radiation against leaf photosynthesis data. Agric. Meteorol. 10,443-453

Varlet-Grancher C. (1982) Analyse du rendement de conversion de l'énergie solaire par un couvert végétal. Thèse doctorat d'Etat, Université Paris-Sud, Centre d'Orsay, $n^{\circ} 2593$ 